\title{
ENTROPY LOCKING
}

\author{
DAVID COSPER AND MICHAL MISIUREWICZ
}

\begin{abstract}
We give a simple proof that in certain one-parameter families of piecewise continuous piecewise linear interval maps with two laps, topological entropy stays constant as the parameter varies.
\end{abstract}

\section{INTRODUCTION}

While continuous interval maps have been thoroughly investigated, the theory of piecewise continuous maps (except piecewise increasing ones) still presents a lot of open questions. Among discontinuous interval maps, the ones with two pieces of continuity/monotonicity (laps) are the simplest ones, so consider such maps, increasing on one lap and decreasing on the other one. To make them even simpler, let us assume that on each lap the map is affine, and in the interior of the lap on which the map is decreasing there is a fixed point. Using an affine conjugacy we can bring such a map to a form where the discontinuity occurs at 0 and the right limit at 0 of the value is 1 . The formula will be then

$$
T_{\lambda, \mu, b}(x)= \begin{cases}1+\lambda x+b & \text { if } \quad x \leq 0 \\ 1-\mu x & \text { if } \quad x \geq 0\end{cases}
$$

where $\lambda, \mu>0$; see Figure 1 .

Observe that unless $b=0$, our maps take two values at 0 . However, this will not create any problems. One should think about the point 0 as sometimes being two points, $0_{-}$and $0_{+}$. Thus, we will write $T_{\lambda, \mu, b}\left(0_{-}\right)$for $\lim _{x{ }^{\prime}} T_{\lambda, \mu, b}(x)$ and $T_{\lambda, \mu, b}\left(0_{+}\right)$for $\lim _{x \searrow 0} T_{\lambda, \mu, b}(x)$. Moreover, if $\lambda$ and $\mu$ are fixed, then we will simply write $T_{b}$ for $T_{\lambda, \mu, b}$.

Define $y_{b}=\max \left\{T_{b}\left(0_{-}\right), T_{b}\left(0_{+}\right)\right\}$and $x_{b}=T_{b}\left(y_{b}\right)$. We want to consider our map on a compact interval instead of the whole real line. The natural candidate for this interval is $\left[x_{b}, y_{b}\right]$. If this interval is invariant for $T_{b}$, then it is the smallest invariant interval. If this interval is not invariant, then the trajectory of $x_{b}$ escapes to $-\infty$, and there is no invariant interval. The necessary and sufficient condition for this interval to be invariant is $T_{b}\left(x_{b}\right) \in\left[x_{b}, y_{b}\right]$. Since always $T_{b}\left(x_{b}\right)<y_{b}$, our condition becomes

$$
T_{b}\left(x_{b}\right) \geq x_{b} \text {. }
$$

While we could translate (2) to inequalities in $\lambda, \mu$ and $b$, we would never use them in that form.

We also want the map to be (eventually) piecewise expanding, so we assume that

$$
\lambda \geq 1 \text { and } \mu>1 \text {. }
$$

Date: November 14, 2016.

2010 Mathematics Subject Classification. 37E05, 37B40.

Key words and phrases. Interval maps, topological entropy, transitivity, maps of constant slope. 


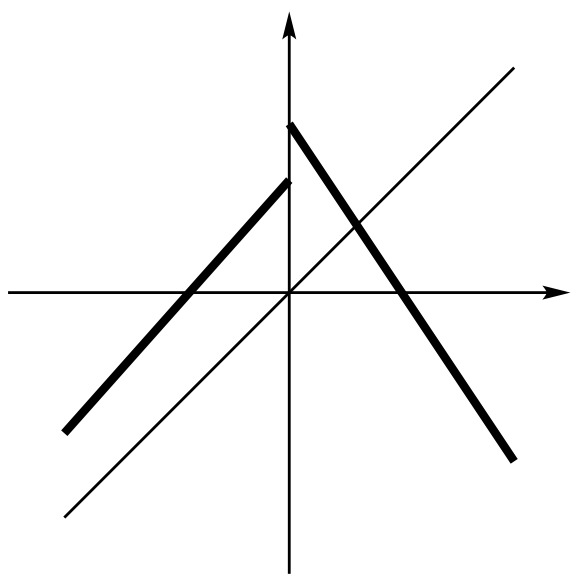

$\mathrm{b}<0$

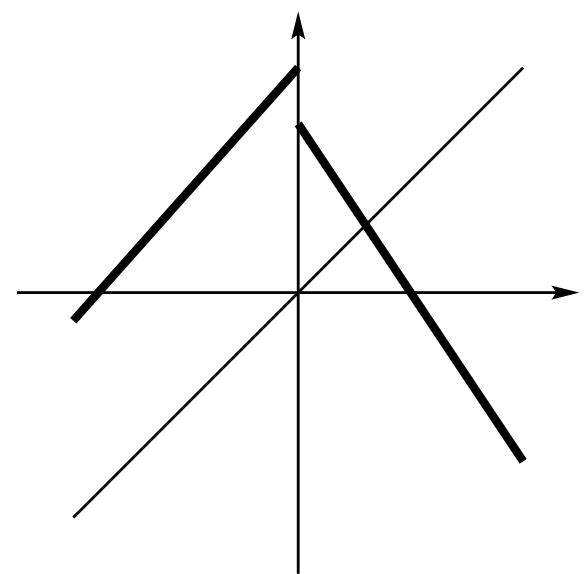

$b>0$

FiguRE 1. The maps $T_{\lambda, \mu, b}$.

However, if in both (2) and (3) we have equalities, then the map on the left lap is the identity. This is a highly degenerate case, so we will assume that

$$
\text { if } T_{b}\left(x_{b}\right)=x_{b} \text { then } \lambda>1
$$

Throughout most of the paper we will consider maps $T_{b}=T_{\lambda, \mu, b}$ satisfying (2), (3) and (4). We will denote the family of those maps by $\mathcal{T}$.

The map $T_{b}$ has a fixed point

$$
z=\frac{1}{1+\mu}
$$

on the right lap. Note that its position does not depend on $b$, so we do not need a subscript $b$ here.

For a piecewise continuous piecewise monotone map $f$ (with the finite number of laps), the usual definition of its topological entropy is

$$
h_{\text {top }}(f)=\lim _{n \rightarrow \infty} \frac{1}{n} \log c_{n},
$$

where $c_{n}$ is the number of laps of $f^{n}$. In [8] it is shown that this agrees with the standard Bowen's definition of topological entropy.

In 2013, V. Botella-Soler, J. A. Oteo, J. Ros and P. Glendinning [5] noticed that for certain values of $\lambda$ and $\mu$ both Lyapunov exponent and topological entropy of $T_{\lambda, \mu, b}$ remain constant as $b$ varies in some interval of values close to 0 , although the kneading sequence varies. In 2014, H. Bruin, C. Carminati, S. Marmi and A. Profeti [3] explained this phenomenon by observing that it is caused by matching with index zero, where for some $k>0$ we have $T_{b}^{k}\left(0_{-}\right)=T_{b}^{k}\left(0_{+}\right)$. In fact, in their definition of matching there is also a similar condition on the derivatives, but we do not need it here (although it is satisfied). 
In this paper we are giving a simple explanation of the matching with index zero phenomenon and of the fact that the topological entropy remains constant (we call this phenomenon entropy locking).

\section{KNEADING THEORY}

Kneading theory is a standard tool for studying maps of the interval. We will use the notation of $[6]$. For $x \in\left[x_{b}, y_{b}\right]$ we define its itinerary $I(x)$ to be the sequence $I_{0}(x) I_{1}(x) I_{2}(x) \ldots$, where

$$
I_{0}(x)= \begin{cases}R & \text { if } x>0, \\ C & \text { if } x=0, \\ L & \text { if } x<0,\end{cases}
$$

and $I_{j}(x)=I_{0}\left(f^{j}(x)\right)$. We adopt the convention that the itinerary terminates if $I_{j}(x)=C$ for some $j$. An arbitrary sequence $\underline{A}$ of $R \mathrm{~s}, L \mathrm{~s}$, and $C$ s will be called admissible if it is either an infinite sequence of $R \mathrm{~s}$ and $L \mathrm{~s}$, or a finite (possibly empty) sequence of $R$ s and $L \mathrm{~s}$ followed by a $C$. Note that all itineraries are admissible.

The length of a sequence $\underline{A}$ of $R$ 's and $L$ 's will be denoted by $|\underline{A}|$, and $\underline{A}$ will be called finite if $|\underline{A}|<\infty$. The parity of a finite sequence $\underline{A}$ of $R$ 's and $L$ 's describes the number of $R$ 's in the sequence. The sequence $\underline{A}$ is even if the number of $R \mathrm{~s}$ is even and odd if the number of $R \mathrm{~s}$ is odd. These sequences can be ordered in the following manner.

Definition 2.1. First we have a natural ordering $L<C<R$. Suppose that $\underline{A}=$ $A_{0} A_{1} \ldots$ and $\underline{B}=B_{0} B_{1} \ldots$ are admissible sequences such that $\underline{A} \neq \underline{B}$. Let $n$ be the first index such that $A_{n} \neq B_{n}$. If the finite sequence

$$
A_{0} A_{1} \ldots A_{n-1}=B_{0} B_{1} \ldots B_{n-1}
$$

is even and $A_{n}<B_{n}$, then $\underline{A}<\underline{B}$. If this finite sequence is odd and $A_{n}<B_{n}$, then $\underline{A}>\underline{B}$.

This is known as the parity-lexicographical ordering and it agrees with the ordering of the points on the interval.

Proposition 2.2. For a map $T_{b} \in \mathcal{T}, I(x)<I(y)$ if and only if $x<y$.

We will not prove this proposition here, since its proof is practically identical to the proof for continuous maps (see, e.g., [6]). The only detail that is different, is that we can use the strict inequalities on both sides of the equivalence. This follows from the fact that our maps have iterates that are piecewise expanding, so different points have different itineraries. Let us state it as a lemma.

Lemma 2.3. For a map $T_{b} \in \mathcal{T}$, there is $n$ such that $T_{b}^{n}$ is expanding on each lap.

Proof. If $\lambda>1$, then $T_{b}$ itself is expanding on each lap. Assume that $\lambda=1$. Then, by (2) and (4), $T_{b}\left(x_{b}\right)-x_{b}>0$, and for each $x \in\left[x_{b}, 0\right)$ we have $T_{b}(x)-x=T_{b}\left(x_{b}\right)-x_{b}$. This means that at least one of the points $T_{b}^{i}(x), 0 \leq i \leq n$, belongs to the right lap of $T_{b}$, provided $n>\left|x_{b}\right| /\left(T_{b}\left(x_{b}\right)-x_{b}\right)$. Therefore, for such $n$ the map $T_{b}^{n}$ is expanding with the constant at least $\mu$ on each lap.

We define the left and right kneading sequences of $T_{b}$ to be $K_{-}\left(T_{b}\right)=I\left(T_{b}\left(0_{-}\right)\right)$ and $K_{+}\left(T_{b}\right)=I\left(T_{b}\left(0_{+}\right)\right)$. 


\section{MAtChing}

We are interested in the conditions under which $T_{b}^{k}\left(0_{-}\right)$and $T_{b}^{k}\left(0_{+}\right)$coincide for some $k$. We start with a simple geometric lemma.

Lemma 3.1. Let $f$ be a map conjugated to $T_{\lambda, \mu, 0} \in \mathcal{T}$ via an orientation preserving affine map. Let $c$ be the turning point of $f$ and let $x<c<y$. Then $f(x)=f(y)$ if and only if

$$
\frac{x-c}{c-y}=\frac{\mu}{\lambda}
$$

Proof. Assume that (7) is satisfied. Then

$$
f(x)-f(c)=\lambda(x-c)=\mu(c-y)=f(y)-f(c),
$$

and therefore $f(x)=f(y)$.

Now assume that $f(x)=f(y)$. Then

$$
\lambda(x-c)=f(x)-f(c)=f(y)-f(c)=\mu(c-y),
$$

and (7) follows.

Now we can prove the main result of this section. In the proof we will be using the notation $\langle x, y\rangle$ for $[x, y]$ if $x<y$ and $[y, x]$ if $y<x$.

Theorem 3.2. Let $T_{b}=T_{\lambda, \mu, b} \in \mathcal{T}$, and let $\underline{A}$ be a finite (possibly empty) sequence of symbols $R$ and $L$. Set $n=|R L \underline{A} C|$. Assume that $K_{-}\left(T_{b}\right)=R L \underline{A} R \ldots$ and $K_{+}\left(T_{b}\right)=R L \underline{A} L \ldots$ Then $K\left(T_{0}\right)=\bar{R} L \underline{A} C$ if and only if $T_{b}^{n+1}\left(0_{-}\right)=T_{b}^{n+1}\left(0_{+}\right)$.

Proof. We use the ideas from the Euclidean geometry. We consider the graph of $T_{b}$, then draw some additional lines, identify similar figures and use proportions.

Thus, consider the graph of $T_{b}$. It consists of two branches. From the assumptions on the kneading sequences it follows that $b \neq 0$. If $b<0$, then the left branch ends lower than the right branch; if $b>0$ then the right branch ends lower than the right one. Extend the lower branch until it crosses the higher one (see Figure 2). This happens at the point $\left(c, T_{b}(c)\right)$, where $1+\lambda c+b=1-\mu c$, so

$$
c=\frac{-b}{\mu+\lambda} .
$$

Now we define a continuous map $f$ of $\left[x_{b}, y_{b}\right]$ to itself by

$$
f(x)= \begin{cases}1+\lambda x+b & \text { if } \quad x \leq c, \\ 1-\mu x & \text { if } \quad x \geq c .\end{cases}
$$

We claim that $f^{i}(c) \notin\langle 0, c\rangle$ for $i=1,2, \ldots, n-1$. Indeed, suppose that $f^{i}(c) \in$ $\langle 0, c\rangle$ for some $i \in[1, n-1]$ and $f^{k}(c) \notin\langle 0, c\rangle$ for all $k \in[1, i-1]$. Then $f^{k}(c)=T_{b}^{k}(c)$ for $k \in[1, i]$. Set $U=\left\langle T_{b}\left(0_{-}\right), T_{b}\left(0_{+}\right)\right\rangle$, and note that $T_{b}(c) \in U$.

Since both $K_{-}\left(T_{b}\right)$ and $K_{+}\left(T_{b}\right)$ begin with $R L$, the interval $U$ lies to the right of the fixed point $z$, while 0 and $c$ are to the left of $z$. Therefore $i \geq 2$.

We have

$$
T_{b}\left(0_{+}\right)-T_{b}(c)=-\mu\left(0-\frac{-b}{\mu+\lambda}\right)=\frac{-\mu b}{\mu+\lambda}
$$




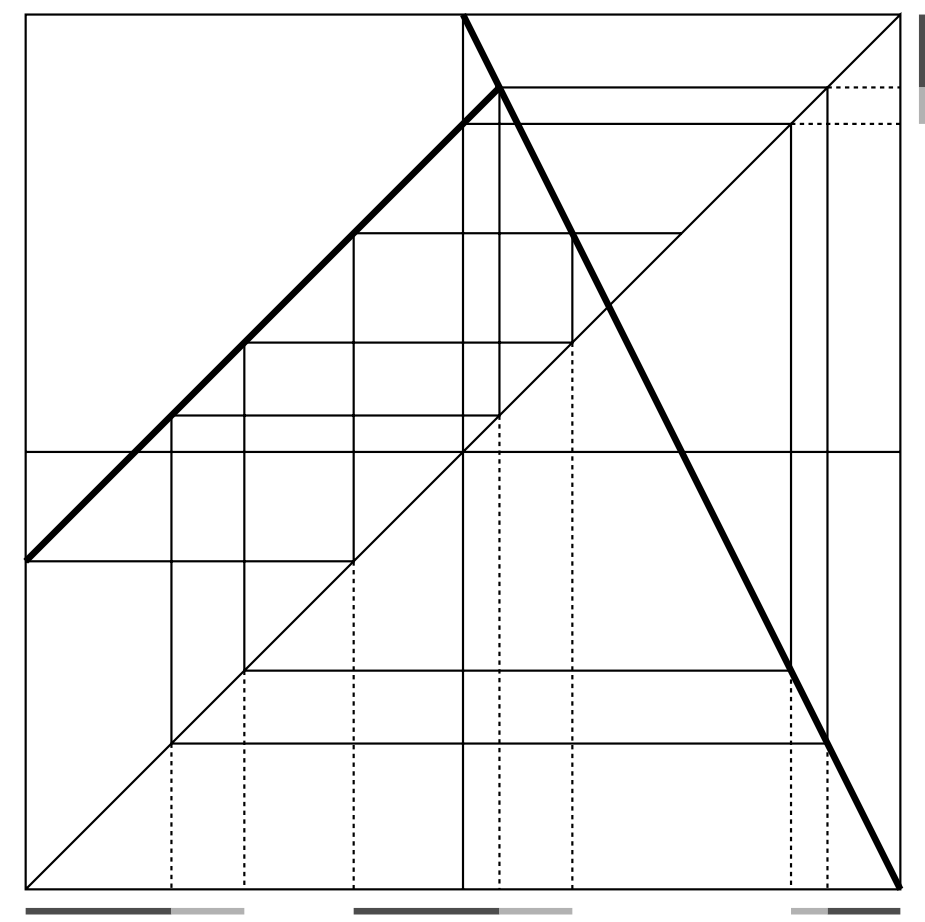

Figure 2. The proof of Theorem 3.2. The proportion of the lengths of the light gray and dark gray intervals stays $\lambda / \mu$.

and

$$
T_{b}(c)-T_{b}\left(0_{-}\right)=\lambda\left(\frac{-b}{\mu+\lambda}-0\right)=\frac{-\lambda b}{\mu+\lambda} .
$$

Since $K_{+}\left(T_{b}\right)$ and $K_{-}\left(T_{b}\right)$ agree on the first $n-1$ places, then $0 \notin T_{b}^{k}(U)$ for $k \leq i$. Therefore, $T_{b}^{i}$ is affine on $U$. Thus, we get

and

$$
\left|T_{b}^{i}\left(0_{+}\right)-T_{b}^{i}(c)\right| \geq \frac{\mu^{2}|b|}{\mu+\lambda}>|c|
$$

$$
\left|T_{b}^{i}(c)-T_{b}^{i}\left(0_{-}\right)\right| \geq \frac{\lambda \mu|b|}{\mu+\lambda}>|c|,
$$

where we get the final inequality because $\mu>1$ and $\lambda \geq 1$. Thus, $K_{+}\left(T_{b}\right)$ and $K_{-}\left(T_{b}\right)$ disagree on the $i-1$ st index, which is a contradiction. This proves that $f^{i}(c) \notin\langle 0, c\rangle$ for $i=1,2, \ldots, n-1$.

It follows from the assumption on kneading sequences that $n \geq 3$, and therefore $f^{2}(c) \notin\langle 0, c\rangle$, so $f^{2}(c)<c$. This implies that the interval $\left[f^{2}(c), f(c)\right]$ is invariant under $f$. Since $f$ has the same slopes as $T_{0}$, then $\left.f\right|_{\left[f^{2}(c), f(c)\right]}$ is conjugate to $T_{0}$ via an orientation preserving affine map. Moreover, it follows from our claim that $f^{i}(c)=T_{b}^{i}(c)$ for all $i \in[0, n]$. Because of the assumptions on kneading sequences, none of the intervals $T_{b}^{i}(U), 0<i<n-1$, contains 0 . Therefore the map $T_{b}^{n-1}$ is affine on $U$. 
From this and from the formulas (9) and (10) it follows that

$$
\frac{T_{b}^{n}\left(0_{+}\right)-T_{b}^{n}(c)}{T_{b}^{n}(c)-T_{b}^{n}\left(0_{-}\right)}=\frac{\mu}{\lambda} .
$$

Assume that $K\left(T_{0}\right)=R L \underline{A} C$. Then $f^{n}(c)=c$. We have $\left|T_{b}^{n}\left(0_{-}\right)-c\right|>|c|$ and $\left|T_{b}^{n}\left(0_{+}\right)-c\right|>|c|$, and thus, $T_{b}^{n}\left(0_{-}\right)$and $T_{b}^{n}\left(0_{+}\right)$are not contained in the interval $\langle 0, c\rangle$. Hence, $T_{b}^{n+1}\left(0_{+}\right)=f\left(T_{b}^{n}\left(0_{+}\right)\right)$and $T_{b}^{n+1}\left(0_{-}\right)=f\left(T_{b}^{n}\left(0_{-}\right)\right)$, so from (11) and Lemma 3.1 we get $T_{b}^{n+1}\left(0_{-}\right)=T_{b}^{n+1}\left(0_{+}\right)$.

Assume now that $T_{b}^{n+1}\left(0_{-}\right)=T_{b}^{n+1}\left(0_{+}\right)$. Then $T_{b}^{n+1}\left(0_{-}\right) \leq \min \left(T_{b}\left(0_{-}\right), T_{b}\left(0_{+}\right)\right)<$ $T_{b}(c)$, so again $T_{b}^{n}\left(0_{-}\right)$and $T_{b}^{n}\left(0_{+}\right)$are not contained in the interval $\langle 0, c\rangle$. By (11) and Lemma 3.1 we get $f^{n}(c)=c$. Since for $i<n$ the point $f^{i}(c)$ is between $T_{b}^{i}\left(0_{-}\right)$and $T_{b}^{i}\left(0_{+}\right)$and both $K_{-}\left(T_{b}\right)$ and $K_{+}\left(T_{b}\right)$ begin with $R L \underline{A}$, and moreover, $f^{i}(c) \notin\langle 0, c\rangle$ for $i=1,2, \ldots, n-1$, we see that the kneading sequence of $f$ also begins with $R L \underline{A}$. Since $f^{n}(c)=c$, the next symbol is $C$. The maps $T_{0}$ and $f$ are conjugate, so their have the same kneading sequences. Therefore, $K\left(T_{0}\right)=R L \underline{A} C$.

Remark 3.3. Suppose that the assumptions of Theorem 3.2 are satisfied. If $b<0$ then $T_{b}\left(0_{-}\right)<T_{b}\left(0_{+}\right)$, so $K_{-}\left(T_{b}\right)<K_{+}\left(T_{b}\right)$. This implies that $\underline{A}$ is even. Similarly, if $b>0$ then $\underline{A}$ is odd.

We can prove a kind of converse to the above remark.

Proposition 3.4. Fix parameters $\lambda \geq 1, \mu>1$, such that $K\left(T_{0}\right)=R L \underline{A C}$. Then if if $\underline{A}$ is even (respectively, odd), there exists $\varepsilon>0$ such that if $b \in(-\varepsilon, 0)$ (respectively, $b \in(0, \varepsilon))$ then the assumptions of Theorem 3.2 are satisfied, and thus, $T_{b}^{n+1}\left(0_{-}\right)=$ $T_{b}^{n+1}\left(0_{+}\right)$.

Proof. If $|b|$ is sufficiently small, then both $K_{-}\left(T_{b}\right)$ and $K_{+}\left(T_{b}\right)$ begin with $R L \underline{A}$. Thus, we have to show that the next symbol is $R$ for $K_{-}\left(T_{b}\right)$ and $L$ for $K_{+}\left(T_{b}\right)$. By making the construction from the proof of Theorem 3.2, we see that $T^{n}\left(0_{-}\right)$and $T^{n}\left(0_{+}\right)$are on the opposite sides of $c$. Moreover, both $\left|T^{n}\left(0_{-}\right)-c\right|$ and $\left|T^{n}\left(0_{+}\right)-c\right|$ are larger than $|c|$, so the $n$th terms of $K_{-}\left(T_{b}\right)$ and $K_{+}\left(T_{b}\right)$ are distinct. Taking into account the order in the set of itineraries (as in Remark 3.3), we get the assertion of the proposition.

\section{TOPOLOGICAL ENTROPY}

Entropy locking refers to intervals of the parameter $b$ where topological entropy of $T_{b}$ remains constant. It turns out that the intervals of parameter $b$ satisfying Theorem 3.2 are intervals with entropy locking.

We need some estimates of the topological entropy for piecewise continuous piecewise monotone interval maps (when using this term, we always assume that the number of pieces is finite). They are known, but they are difficult to find in the literature. Since the proofs are simple, we provide them here.

For a piecewise continuous piecewise monotone interval map $f$ we will say that $\alpha$ is an anti-Lipschitz constant if for every $x, y$ from the same lap we have $|f(x)-f(y)| \geq$ $\alpha|x-y|$. In particular, a map with an anti-Lipschitz constant larger than 1 is piecewise expanding. 
An $s$-horseshoe for $f$ is an interval $J$ and a partition $D=\left\{J_{1}, \ldots, J_{s}\right\}$ of $J$ into $s$ subintervals such that $J \subset f\left(J_{i}\right)$ and $f$ is continuous and monotone on each $J_{i}$. The following theorem was proved in [8].

Theorem 4.1. If $f$ is a piecewise continuous piecewise monotone interval map, then for every $\varepsilon>0$ there exist $n$ and $s$, such that $f^{n}$ has an $s$-horseshoe and $(1 / n) \log s>$ $h_{\text {top }}(f)-\varepsilon$.

Now we can prove the promised estimates.

Theorem 4.2. If $f$ is a piecewise continuous piecewise monotone interval map with an anti-Lipschitz constant $\alpha$ and a Lipschitz constant $\beta$, then $\log \alpha \leq h_{\mathrm{top}}(f) \leq \log \beta$.

Proof. We use formula (5). If the interval on which $f$ is acting has length $\gamma$, then the length of each lap of $f^{n}$ is not larger than $\gamma / \alpha^{n}$. Therefore $c_{n} \geq \alpha^{n}$, and thus, $h_{\text {top }}(f) \geq \log \alpha$.

Take $\varepsilon>0$. By Theorem 4.1, there exist $n$ and $s$, such that $f^{n}$ has an $s$-horseshoe and $(1 / n) \log s>h_{\text {top }}(f)-\varepsilon$. Let an interval $J$ and a partition $D=\left\{J_{1}, \ldots, J_{s}\right\}$ be this horseshoe. Then the length of each $J_{i}$ is at least the length of $J$ divided by $\beta^{n}$. Therefore, $s \leq \beta^{n}$, and hence, $\log \beta>h_{\text {top }}(f)-\varepsilon$. Since $\varepsilon>0$ was arbitrary, we get $h_{\text {top }}(f) \leq \log \beta$.

From this theorem and Lemma 2.3, we get immediately the following corollary.

Corollary 4.3. All maps from $\mathcal{T}$ have strictly positive topological entropy.

Any map $T_{\lambda, \lambda, b} \in \mathcal{T}$ has both anti-Lipschitz and Lipschitz constants equal to $\lambda$. Therefore we get immediately another corollary to Theorem 4.2.

Corollary 4.4. If $T_{\lambda, \lambda, b} \in \mathcal{T}$, then its topological entropy is $\log \lambda$.

Now we are ready to prove the main result of this section. We will refer to piecewise continuous piecewise affine interval maps with the absolute value of the derivative constant, as maps of constant slope. In $\mathcal{T}$, these are maps of the form $T_{\lambda, \lambda, b}$.

We will be using often a certain long assumption, so it makes sense to give it a short name.

Definition 4.5. We will say that $T_{b}$ satisfies the kneading assumption if $T_{b}=T_{\lambda, \mu, b} \in$ $\mathcal{T}$ and there exists a finite (possibly empty) sequence $\underline{A}$ of symbols $R$ and $L$, such that $K\left(T_{0}\right)=R L \underline{A} C, K_{-}\left(T_{b}\right)=R L \underline{A} R \ldots$, and $K_{+}\left(T_{b}\right)=R L \underline{A} L \ldots$

Theorem 4.6. Assume that $T_{b}$ satisfies the kneading assumption and is topologically conjugate to a map of constant slope. Then $h_{\mathrm{top}}\left(T_{b}\right)=h_{\mathrm{top}}\left(T_{0}\right)$.

Proof. By the assumption, $T_{\lambda, \mu, b}$ is conjugate to $T_{\alpha, \alpha, d}$ for some $\alpha$ and $d$. By Corollary 4.4,

$$
\log \alpha=h_{\mathrm{top}}\left(T_{\alpha, \alpha, d}\right)=h_{\mathrm{top}}\left(T_{\lambda, \mu, b}\right) .
$$

Set $n=|R L \underline{A} C|$. From Theorem 3.2 it follows that $T_{\lambda, \mu, b}^{n+1}\left(0_{+}\right)=T_{\lambda, \mu, b}^{n+1}\left(0_{-}\right)$. Hence, $T_{\alpha, \alpha, d}^{n+1}\left(0_{+}\right)=T_{\alpha, \alpha, d}^{n+1}\left(0_{-}\right)$. Since the kneading sequences are preserved by a conjugacy, the left and right kneading sequences of $T_{\alpha, \alpha, d}$ are $K_{-}\left(T_{\alpha, \alpha, d}\right)=R L \underline{A} R \ldots$ and $K_{+}\left(T_{\alpha, \alpha, d}\right)=R L \underline{A} L \ldots$, respectively. Thus, we can use Theorem 3.2 again, and we 
get $K\left(T_{\alpha, \alpha, 0}\right)=R L \underline{A} C$. For unimodal maps the topological entropy is determined by the kneading sequence, and therefore

$$
h_{\mathrm{top}}\left(T_{\lambda, \mu, 0}\right)=h_{\mathrm{top}}\left(T_{\alpha, \alpha, 0}\right) .
$$

By Corollary 4.4,

$$
h_{\text {top }}\left(T_{\alpha, \alpha, 0}\right)=\log \alpha .
$$

From (12), (13) and (14) we get $h_{\mathrm{top}}\left(T_{\lambda, \mu, 0}\right)=h_{\mathrm{top}}\left(T_{\lambda, \mu, b}\right)$.

\section{TRANSITIVITY}

While Theorem 4.6 is quite strong, it contains an assumption that may be not easy to verify in concrete situations. Namely, we assume that $T_{b}$ is topologically conjugate to a map of constant slope. In this section we will try to replace this assumption by weaker ones, which are easier to verify.

The first idea is to assume that $T_{b}$ is topologically transitive. The following theorem can be found for instance in [2].

Theorem 5.1. If $f$ is a piecewise continuous piecewise monotone topologically transitive interval map with topological entropy $\log \beta>0$, then it is topologically conjugate to a map of constant slope $\beta$.

In view of this theorem and Corollary 4.3, we get the following corollary to Theorem 4.6.

Corollary 5.2. Assume that $T_{b}$ satisfies the kneading assumption and is topologically transitive. Then $h_{\mathrm{top}}\left(T_{b}\right)=h_{\mathrm{top}}\left(T_{0}\right)$.

We will further improve this corollary, by replacing the assumption that $T_{b}$ is topologically transitive by another assumption, which is maybe a little weaker, but easier to check. This assumption will be

$$
T_{\lambda, \mu, 0}\left(x_{0}\right)<z .
$$

It can be easily written as an inequality on parameters

$$
\lambda+\mu<\lambda \mu^{2} .
$$

It is known that it is equivalent to $T_{\lambda, \mu, 0}$ being totally transitive; however, we will not use this fact. We will say that $T_{b}=T_{\lambda, \mu, b}$ satisfies (15) if $T_{0}=T_{\lambda, \mu, 0}$ satisfies it.

Definition 5.3. The set $\mathcal{T}_{\mathrm{KAT}}$ is the set of all maps $T_{b}$ satisfying both the kneading assumption and (15).

Lemma 5.4. Assume that $T_{b} \in \mathcal{T}_{\mathrm{KAT}}$. Then

$$
T_{b}(1-\mu) \leq 1 \text {. }
$$

Proof. If $b \leq 0$, then $y_{b}=1$, so (17) holds. Assume that $b>0$. If $T_{b}(1-\mu)>1$, then $K_{+}\left(T_{b}\right)=R L R L \ldots$ By the kneading assumption, $K_{-}\left(T_{b}\right)=R L R \ldots$ We have $T_{b}\left(x_{b}\right)=1+\lambda(1-\mu-\mu b)+b$ and $T_{0}\left(x_{0}\right)=1+\lambda(1-\mu)$. Since $b<\lambda \mu b$, we get $T_{b}\left(x_{b}\right)<T_{0}\left(x_{0}\right)$. By this and (15), $T_{b}\left(x_{b}\right)<z$, so the next term in $K_{-}\left(T_{b}\right)$ is $R$. Thus, by the kneading assumption, $K\left(T_{0}\right)=R L R C$. Then $1-\mu(1+\lambda-\lambda \mu)=T_{0}^{4}(0)=0$, so $\lambda=1 / \mu<1$, a contradiction. Thus, (17) holds. 
Lemma 5.5. Assume that $T_{b} \in \mathcal{T}_{\mathrm{KAT}}$. Let $U$ be an interval containing $z$. Then

$$
\bigcup_{i=0}^{\infty} T_{b}^{i}(U)=\left[x_{b}, y_{b}\right] \text {. }
$$

Proof. Suppose first that $b \leq 0$. Then $\left[x_{b}, y_{b}\right]=[1-\mu, 1]$. Since the interval $U$ contains $z$, then all sets $T_{b}^{i}(U)$ must contain $z$ as well. Moreover, $\mu>1$, so the length of $T_{b}^{i}(U)$ is expanding exponentially with $i$ until we reach an $m$ such that $T_{b}^{m}(U)$ contains $[z, 1]$. Therefore $T_{b}([z, 1])=[1-\mu, z] \subset T_{b}^{m+1}(U)$. Hence, $T_{b}^{m}(U) \cup T_{b}^{m+1}(U)=\left[x_{b}, y_{b}\right]$.

Now assume that $b>0$. By Lemma 5.4, (17) holds. As in the case $b \leq 0$, we get $T_{b}^{m}(U) \cup T_{b}^{m+1}(U) \supset[1-\mu, 1]$ for some $m$. Since $T_{b}(1-\mu) \leq 1$, the interval $T_{b}([1-\mu, 0])$ contains $\left[1, y_{b}\right]$. Since $T_{b}\left(\left[1, y_{b}\right]\right)=\left[x_{b}, 1-\mu\right]$, we get $T_{b}^{m}(U) \cup T_{b}^{m+1}(U) \cup$ $T_{b}^{m+2}(U) \cup T_{b}^{m+3}(U)=\left[x_{b}, y_{b}\right]$.

Theorem 5.6. Assume that $T_{b} \in \mathcal{T}_{\mathrm{KAT}}$. Then $T_{b}$ is topologically transitive.

Proof. Let $U$ be an open subinterval of $\left[x_{b}, y_{b}\right]$. We will show that $V=\bigcup_{i=0}^{\infty} T_{b}^{i}(U)$ is dense in $\left[x_{b}, y_{b}\right]$. Since $\mu>1$, the length of $T_{b}^{n}(U)$ increases exponentially with $n$. Thus, there exists $k$ such that $0 \in T_{b}^{k}(U)$. Therefore, $V$ contains an interval containing 0 . Let $W$ be the largest such interval contained in $V$. We can write $W=W_{L} \cup W_{R}$, where $W_{L}=\{x \in W: x \leq 0\}$ and $W_{R}=\{x \in W: x \geq 0\}$. Since $V$ is invariant and $\mu>1$, then, by the same reason as for $U$, it must happen that 0 belongs to the interior of $T_{b}^{m}\left(W_{L}\right)$ and $T_{b}^{n}\left(W_{R}\right)$ for some positive integers $m$ and $n$. If $m$ and $n$ are minimal such integers, then $T_{b}^{m}\left(W_{L}\right)$ and $T_{b}^{n}\left(W_{R}\right)$ are intervals, and therefore they are contained in $W$.

Suppose that $V$ is not dense. We claim that then $m \geq 2$ and $n \geq 2$. In view of Lemma 5.5, in order to prove the claim, it is enough to show that if $m$ or $n$ is 1 , then $z \in W$.

Assume first that $b<0$. If $m=1$, then $T_{b}\left(W_{L}\right) \subset W$ and in particular $T_{b}\left(0_{-}\right) \in W$. Therefore the interval $\left[0, T_{b}\left(0_{-}\right)\right]$is contained in $W$. We claim that $T_{b}\left(0_{-}\right) \geq z$. Indeed, if $T_{b}\left(0_{-}\right)<z$, then $K_{-}\left(T_{b}\right)$ starts with $R R$, which is impossible by the kneading assumption, and this proves the claim. Therefore, $z \in\left[0, T_{b}\left(0_{-}\right)\right]$. If $n=1$, then $T_{b}\left(W_{R}\right) \subset W$, and in particular $T_{b}\left(0_{+}\right)=1 \in W$. Thus, $z \in[0,1] \subset W$.

Now assume that $b>0$. If $m=1$, then $T_{b}\left(W_{L}\right) \subset W$ and in particular $T_{b}\left(0_{-}\right)=$ $1+b \in W$. Thus, $z \in[0,1+b] \subset W$. If $\mathrm{n}=1$, then $T_{b}\left(W_{R}\right) \subset W$ and it follows that $z \in[0,1] \subset W$. This completes the proof of the claim.

By our choice of $m$ and $n, T_{b}^{m}$ is affine on $W_{L}$ and $T_{b}^{n}$ is affine on $W_{R}$. Additionally, since $T_{b}\left(0_{-}\right)>0, T_{b}\left(0_{+}\right)>0$ and $m, n \geq 2$, we have $I(x)=L R \ldots$ for every $x \in W_{L}$ and $I(x)=R R \ldots$ for every $x \in W_{R}$.In such a way, we get lower bounds on the lengths of $T_{b}^{m}\left(W_{L}\right)$ and $T_{b}^{n}\left(W_{R}\right)$ :

$$
\begin{aligned}
\lambda \mu\left|W_{L}\right| & \leq\left|T_{b}^{m}\left(W_{L}\right)\right|, \\
\mu^{2}\left|W_{R}\right| & \leq\left|T_{b}^{n}\left(W_{R}\right)\right| .
\end{aligned}
$$

We also know that $T_{b}^{m}\left(W_{L}\right) \subset W$ and $T_{b}^{n}\left(W_{R}\right) \subset W$, so from (18) we get

$$
\begin{aligned}
& \lambda \mu\left|W_{L}\right| \leq|W|, \\
& \mu^{2}\left|W_{R}\right| \leq|W| .
\end{aligned}
$$


We add the first inequality in (19) multiplied by by $\mu$ to the second one multiplied by $\lambda$, and taking into account that $\left|W_{L}\right|+\left|W_{R}\right|=|W|$, we get

$$
\lambda \mu^{2}|W| \leq(\lambda+\mu)|W|,
$$

which contradicts (16) (which, as we noticed, is equivalent to (15)). This completes the proof.

Now from Corollary 5.2 and Theorem 5.5 we get an improved corollary.

Corollary 5.7. Assume that $T_{b} \in \mathcal{T}_{\mathrm{KAT}}$. Then $h_{\mathrm{top}}\left(T_{0}\right)=h_{\mathrm{top}}\left(T_{b}\right)$.

\section{BEYOND TRANSITIVITY}

Theorem 5.6 gives sufficient conditions for transitivity of $T_{b}=T_{\lambda, \mu, b}$. The assumption of this theorem is that $T_{b} \in \mathcal{T}_{\mathrm{KAT}}$, that is, that $T_{b}$ satisfies the kneading assumption and satisfies 15. The examples in this section will show that both assumptions are essential.

First, we establish a necessary condition for transitivity.

Lemma 6.1. Suppose $T_{b} \in \mathcal{T}$. If $z \notin T_{b}\left(\left[x_{b}, 0\right]\right)$, then $T_{b}$ is not transitive.

Proof. Let $\varepsilon$ be sufficiently small so that $(z-\varepsilon, z+\varepsilon) \cap T_{b}\left(\left[x_{b}, 0\right]\right)=\emptyset$. Since $\mu>1, z$ is repelling, and therefore $T_{b}^{-1}((z-\varepsilon, z+\varepsilon)) \subset(z-\varepsilon, z+\varepsilon)$. Hence, if $V$ is an open interval such that $(z-\varepsilon, z+\varepsilon) \cap V=\emptyset$, then $T_{b}^{n}(V) \cap(z-\varepsilon, z+\varepsilon)=\emptyset$ for all $n$. Thus, $T_{b}$ is not transitive.

Example 6.2. Set $\lambda=1$ and find $\mu$ such that the kneading sequence of $T_{0}=T_{\lambda, \mu, 0}$ is $R L R R R C$. Elementary computations show that $\mu$ is the real solution of the equation $\mu^{3}-\mu^{2}-1=0(\mu \approx 1.46557)$. We can deduce from the kneading sequence that $T_{0}\left(x_{0}\right)>z$, so $T_{0}$ does not satisfy $(15)$. Moreover, $T_{b}\left(x_{b}\right)>z$ for sufficiently small $b$. It follows from Proposition 3.4 that $T_{b}$ satisfies the kneading assumption for sufficiently small $b>0$. Hence, for $b>0$ sufficiently small, $T_{b}$ satisfies the kneading assumption, but not (15), and is not transitive.

Example 6.3. Set $\lambda=1$ and $\mu=2$. Then $K\left(T_{0}\right)=R L C$ and $T_{0}\left(x_{0}\right)<z$. Therefore, $T_{b}$ satisfies (15) for any $b$. However, for $b=-\frac{3}{4}$ we have $T_{b}\left(0_{-}\right)<z$, so by Lemma 6.1 , $T_{b}$ is not transitive. In particular, it cannot satisfy the kneading assumption.

We will show that also the topological entropies of $T_{0}$ and $T_{b}$ are different. Both maps are Markov. For $T_{0}$, the Markov partition consists of two intervals, and its Markov graph is as in Figure 3. Therefore, the topological entropy of $T_{0}$ is the logarithm of the positive solution of the equation $x^{2}-x-1=0$, that is, the logarithm of the golden ratio $\phi=\frac{1+\sqrt{5}}{2} \approx 1.618$.

$$
I_{1} \longleftrightarrow I_{2}
$$

Figure 3. Markov graph for $T_{0}$.

For the map $T_{b}$, we have $T_{b}^{6}\left(0_{+}\right)=0$ and $T_{b}^{3}\left(0_{-}\right)=0$. A Markov partition $\mathcal{P}$ of $\left[x_{b}, y_{b}\right]=[-1,1]$ is given by the orbits of $0_{+}$and $0_{-}$. We shall denote the intervals of this partition by $J_{i}$, as illustrated in Figure 4. 


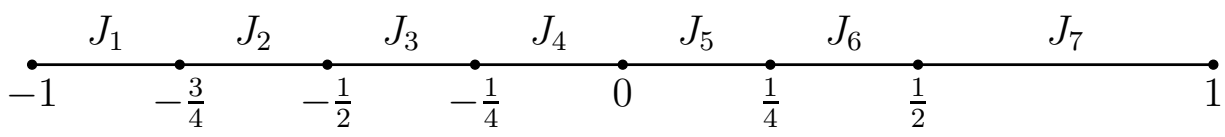

FiguRE 4. Markov partition $\mathcal{P}$ for $T_{b}$.

The Markov graph for $T_{b}$ with the partition $\mathcal{P}$ is presented in Figure 5. One can find easily its entropy using the rome method (see [4] or [1]). It is equal to the logarithm of the positive solution of the equation $x^{6}-x^{3}-x^{2}-x-1=0$, that is, approximately $\log 1.3803$. Hence, $h_{\mathrm{top}}\left(T_{0}\right) \neq h_{\mathrm{top}}\left(T_{b}\right)$ for $b=-3 / 4$. A reader, that does not believe in approximate values, can check that

$$
x^{6}-x^{3}-x^{2}-x-1=\left(x^{4}+x^{3}+2 x^{2}+2 x+3\right)\left(x^{2}-x-1\right)+(4 x+2),
$$

so $\phi^{6}-\phi^{3}-\phi^{2}-\phi-1=4 \phi+2>0$.

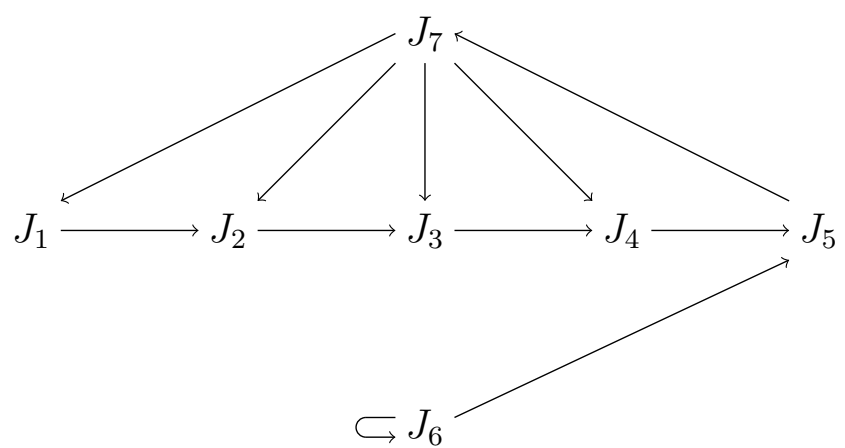

Figure 5. Markov graph associated to the partition $\mathcal{P}$.

Remember that the reason we started to consider transitivity of $T_{b}$ was that we do not know any other simple way of verifying that $T_{b}$ is conjugate to a map of constant slope. However, the maps $T_{0} \in \mathcal{T}$ are known to be conjugate to maps of constant slope (this basically follows from [7] and [6], although it is not stated explicitly there). Thus, we can state the following conjecture.

Conjecture 6.4. Every $T_{b} \in \mathcal{T}$ is topologically conjugate to a map of constant slope.

If this conjecture is true, then by Theorem 4.6 every map $T_{\lambda, \mu, b} \in \mathcal{T}$ satisfying the kneading assumption would have the same topological entropy as $T_{\lambda, \mu, 0}$.

\section{REFERENCES}

[1] Ll. Alsedà, J. Llibre and M. Misiurewicz, Combinatorial Dynamics and Entropy in Dimension One, 2nd edition, Advanced Series in Nonlinear Dynamics 5, World Scientific, Singapore, 2000.

[2] Ll. Alsedà and M. Misiurewicz, Semiconjugacy to a map of a constant slope, Discrete Contin. Dynam. Sys. B 20 (2015), 3403-3413.

[3] H. Bruin, C. Carminati, A. Profeti and S. Marmi, Matching for discontinuous interval maps, talk

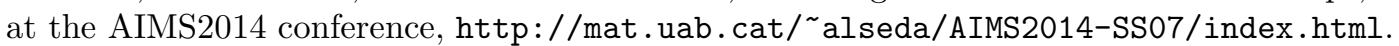

[4] L. Block, J. Guckenheimer, M. Misiurewicz and L.-S. Young, Periodic points and topological entropy of one dimensional maps, in "Global Theory of Dynamical Systems", (Lecture Notes in Math. 819), Springer, Berlin 1980, pp. 18-34. 
[5] V. Botella-Soler, J. A. Oteo, J. Ros and P. Glendinning, Lyapunov exponent and topological entropy plateaus in piecewise linear maps, J. Phys. A 46 (2013), 125101, 26 pp.

[6] P. Collet and J.-P. Eckmann, Iterated maps on the interval as dynamical systems, Progress in Physics 1, Birkhäuser, Boston, Mass., 1980.

[7] M. Misiurewicz and E. Visinescu, Kneading sequences of skew tent maps, Ann. Inst. H. Poincaré, Probab. Stat. 27 (1991), 125-140.

[8] M. Misiurewicz and K. Ziemian, Horseshoes and entropy for piecewise continuous piecewise monotone maps, in "From phase transitions to chaos", World Scientific, Singapore 1992, pp. 489-500.

Department of Mathematical Sciences, IUPUi, 402 N. Blackford Street, IndiANAPOLIS, IN 46202

E-mail address: dcosper@umail.iu.edu

Department of Mathematical Sciences, IUPUi, 402 N. Blackford Street, IndiANAPOLIS, IN 46202

E-mail address: mmisiure@math.iupui.edu 